\title{
The role of direct renin inhibitors in the treatment of the hypertensive diabetic patient
}

\author{
Graziano Riccioni
}

\begin{abstract}
Hypertensive patients with diabetes exhibit an increased risk for cardiovascular complications, such as acute coronary syndrome, stroke, heart failure and chronic kidney disease (CKD). These two chronic diseases are linked to a high rate of morbidity and mortality and for this reason it is important for the clinician to recognize the need for effective treatment of hypertension, which can require combination therapy to achieve blood pressure (BP) goals. Direct renin inhibitors (DRIs) may be useful in combination with angiotensin-converting enzyme inhibitors (ACEIs) or angiotensin II receptor blockers (ARBs) as they provide a more complete blockade of the renin-angiotensin-aldosterone system (RAAS), effectively suppressing residual angiotensin II production and the counter-regulatory increase in plasma renin activity observed in patients receiving monotherapy with ACEls or ARBs. Some questions regarding the action of aliskiren in cardiovascular and renal disorders are open. In particular, the combination therapy of aliskiren and a RAAS blocker in diabetic hypertensive patients with CKD is controversial. Several published studies demonstrated that aliskiren is suitable for once-daily administration and its antihypertensive effect is comparable or superior to that of other antihypertensive agents at recommended doses, with a good tolerability profile. At the moment the association with ACEls and ARBs is not recommended in patients with type 2 diabetes mellitus (T2DM) and renal impairment even if a recent published open-label study of low-dose aliskiren (150 mg/daily) in association with ACEls or ARBs has demonstrated a good tolerability profile without the adverse events found in other studies. This review provides a brief overview of RAAS blocking, in particular the rationale and clinical evidence supporting the use of the DRI aliskiren, in high-risk patients with T2DM.
\end{abstract}

Keywords: aliskiren, diabetes, diabetic nephropathy, hypertension, RAAS, renin

\section{Introduction}

Diabetes and cardiovascular disease (CVD) represent two important and devastating diseases that continue to take a toll on millions of patients worldwide. Scientific evidence demonstrates that these two diseases are integrally related, in particular with regard to the underlying pathophysiological processes and ultimate clinical outcomes [Perk et al. 2012]. Patients with diabetes are two to four times more likely to develop CVD than those without diabetes, $80 \%$ of patients with type 2 diabetes mellitus (T2DM) will develop CVD, and more than $60 \%$ of these patients will die from CVD [Buse et al. 2012].
Hypertension is the primary attributable risk factor for mortality, is reported in over two-thirds of patients with T2DM, and its development coincides with the development of hyperglycemia. Many pathophysiological mechanisms underlie this association. Of these mechanisms, insulin resistance in the nitric-oxide (NO) pathway, the stimulatory effect of hyperinsulinemia on sympathetic drive, smooth muscle growth, sodium-fluid retention, and the excitatory effect of hyperglycemia on the renin-angiotensin-aldosterone system (RAAS) are involved. In patients with diabetes, hypertension confers an enhanced risk of CVD [Ferrarini and Cushman, 2012]. A RAAS system
Ther Adv Endocrinol

Metab

(2013) 4(5) 139-145

DOI: $10.1177 /$

2042018813490779

(c) The Author(s), 2013. Reprints and permissions: http://www.sagepub.co.uk/ journalsPermissions.nav
Correspondence to: Graziano Riccioni, MD, PhD Intensive Cardiology Care Unit, San Camillo de Lellis Hospital, Via G. De Rogatis, 12, 71016 San Severo (FG), Italy griccioniahotmail.com 
blocker combined with a thiazide-type diuretic might be the best initial antihypertensive regimen for most people with diabetes. In general, the positive effects of antihypertensive drugs on cardiovascular outcomes outweigh the negative effects of antihypertensive drugs on glucose metabolism.

Despite the widespread impact of hypertension and its negative outcomes, blood pressure (BP) control in patients with T2DM remains suboptimal. Approximately $25 \%$ of adults who have hypertension are unaware of their condition, and more than $40 \%$ of patients receiving treatment do not achieve the modest goal of $<140 / 90 \mathrm{mmHg}$ [Egan et al. 2012]. The main classes of antihypertensive medications are thiazide-type diuretics, calcium channel blockers, $\beta$-blockers, angiotensin-converting enzyme inhibitors (ACEIs), and angiotensin II receptor blockers (ARBs). Agents in the latter two classes target the RAAS, which plays a significant role in regulating blood volume, arterial pressure, and cardiac and vascular function. ACEIs and ARBs are the second and fifth most commonly prescribed treatments for hypertension [Ma and Stafford, 2008]. RAAStargeted therapies also include direct renin inhibitors (DRIs) [Riccioni, 2011].

The pharmacological inhibition of the RAAS can be obtained through three different basic mechanisms: (1) inhibition of angiotensin II (Ang II) generation from angiotensin I (Ang I), achieved through inhibition of angiotensin-converting enzyme (ACE); (2) inhibition of the action of Ang II at the level of its receptor(s); and (3) inhibition of Ang I generation from angiotensinogen (Ang) obtained by direct inhibition of renin [Cagnoni et al. 2010]. The rationale for the use of these three drug classes has steadily expanded beyond their proven efficacy as antihypertensive agents. In particular, they have been shown to provide special advantages in three groups of patients: those with heart failure, coronary ischemia, or nephropathy [Giovanna et al. 2012; Von Lueder and Krum, 2012].

ACEIs block the action of ACE, a bivalent dipeptidyl carboxyl metallo-peptidase that cleaves the C-terminal dipeptide from Ang I and bradykinin. The use of ACEIs in the treatment of hypertension has steadily expanded as they have been shown to provide special advantages in a large group of hypertensive patients. ARBs displace Ang II from its specific angiotensin type $1\left(\mathrm{AT}_{1}\right)$ receptor, antagonizing all of its known effects and resulting in both a dose-dependent fall in peripheral resistance and little change in heart rate or cardiac output. As a consequence of this competitive displacement, the circulating level of Ang II increases and, at the same time the blockade of the renin-angiotensin mechanism is more complete, including any Ang II that is generated through pathways that do not involve ACE [Mentz et al. 2012]. An important and obvious difference between ARBs and ACEIs is the absence of an increase in kinin levels that may be responsible for some of the beneficial effects of ACEIs and their side effects. The compounds that block the vital stages of the RAAS cascade, such as ACEIs, ARBs, and aldosterone receptor antagonists, importantly, have extended our treatment options. However, the positive therapeutic effects of these compounds also have certain negative consequences. Administration of ACEIs and ARBs interrupts physiological feedback for renal renin release and leads to reactive elevation of circulating active renin and greater production of Ang I and Ang II, with subsequent return of aldosterone secretion to the pretreatment levels ('escape' phenomenon). These possible adverse effects of the intermediary products of incomplete RAAS blockade leading to organ complications have facilitated efforts to develop compounds blocking the initial stages of the renin-angiotensin cascade such aliskiren, which reduces plasma renin activity (PRA) and neutralizes hydrochlorothiazideinduced RAAS activation [Cagnoni et al. 2010].

\section{Aliskiren: the first DRI}

Aliskiren is the first representative of a new class of oral DRIs with a high binding affinity for renin [Rahuel et al. 2000]. In healthy humans, aliskiren in doses between 40 and $640 \mathrm{mg}$ exerts a dosedependent reduction in PRA, Ang I, and Ang II levels. The molecule is superior in reducing PRA compared with ARBs. Again, aliskiren at a dose of $300 \mathrm{mg}$ decreases PRA in hypertensive patients by $\sim 50-80 \%$ and reduces PRA and plasma levels of Ang I and Ang II for 48 hours [Campbell, 2008; Bruckner, 2007]. Following oral administration, peak plasma concentrations of aliskiren are reached within 1-3 hours [Vaidyanathan et al. 2006]. The plasma half-life of aliskiren in humans shows a slow terminal elimination at $23-70$ hours and $\sim 47-51 \%$ of aliskiren is bound by plasma proteins in humans, independent of the concentration. Based on in vitro studies, the major enzyme responsible for its metabolism appears to be cytochrome P450 (CYP3A4). Aliskiren does not inhibit the CYP450 
isoenzymes (CYP1A2, CYP2C8, CYP2C19, CYP2D6, CYP2E1, and CYP3A), and the main elimination route of aliskiren is via feces in its unmetabolized form. Approximately a quarter of the absorbed dose also appears in the urine as unchanged compound; the pharmacokinetic and pharmacodynamic differences of aliskiren between Europeans and Japanese are minimal and no clinically important pharmacokinetic differences were observed between patients with T2DM and normal population: the half-life of this drug was 40 and 44 hours in healthy subjects and patients with diabetes, respectively [Waldemeier et al. 2007; Wuerzner and Azizi, 2008].

Aliskiren is well tolerated by all age groups, including the elderly, and there are no indications to change the recommended dose of aliskiren in patients with hepatic and renal insufficiency because the peak concentration, area under the curve (AUC), and half-life were only slightly greater in patients with hepatic dysfunction. All agents that inhibit the RAAS activate the negative feedback loop that leads to a compensatory increase in plasma renin concentration. When this increase occurs during treatment with ACEIs and ARBs, the result is increased levels of PRA but during treatment with aliskiren, the effect of increased renin levels is blocked, so the levels of Ang I and Ang II, as well as PRA, are all reduced [Bruckner, 2007]. The clinical trials do not report any major adverse effects of aliskiren compared with ARBs or ACEs, being generally well tolerated by all patients. The most common adverse events of aliskiren are diarrhea, headache, nasopharyngitis, dizziness, fatigue, back pain, gastrointestinal disorders, rash, and renal stones [Oparil et al. 2007; Cheng, 2008]. Because aliskiren directly inhibits the RAAS, theoretically adverse events such as coughing and angioedema may also occur; as a matter of fact, few cases of edema involving the face, lips, tongue, hands, and whole body have been reported to involve more than $1 \%$ of patients treated with aliskiren, which also occur at a similar or greater extent in patients receiving placebo [Riccioni et al. 2009]. Aliskiren has the same contraindications as ACEIs and $\mathrm{ARBs}$, including hypersensitivity reactions to the molecule, pregnancy, and bilateral renal-artery stenosis [Angeli et al. 2012].

\section{The treatment of the hypertensive diabetic patient}

Hypertension is a major modifiable risk factor for cardiovascular morbidity and mortality in patients with T2DM. Lowering BP to $135 / 85$ $\mathrm{mmHg}$ is the main goal of treatment of diabetic patients. A nonpharmacologic approach is recommended in these patients. If BP levels remain above the target despite nonpharmacologic treatment, drug therapy should be initiated. Blockers of the RAAS represent the first-line therapy and the cornerstone of the antihypertensive drug arsenal; however, in most diabetic patients, combination therapy is required [Hsueh and Wyne, 2011]. For this class of patients, a combination of RAAS blocker and calcium channel antagonists are the combination preferred by the treating physician [Volpe et al. 2011]. Often three or even four drugs are needed. Treatment should be individualized according to concomitant risk factors and diseases and depending on the age and hemodynamic and laboratory parameters of the patient [Grossmann and Messerli, 2011].

Numerous clinical studies have demonstrated that hypertension plays a major role in accelerating the occurrence and progression of vascular complications associated with type 1 and type 2 diabetes. Risks for CVD, renal disease, retinopathy, and neuropathy increase significantly when hypertension occurs in association with diabetes [Sowers et al. 2001]. Diabetic nephropathy is the leading cause of kidney disease, affecting $\sim 40 \%$ of those with type 1 or type 2 diabetes [Gross et al. 2005]. Proteinuria occurs in $15-40 \%$ of patients with type 1 diabetes and in $5-20 \%$ of patients with T2DM [Anothaisintawee et al. 2009]. In patients with T2DM, microalbuminuria or overt albuminuria is a stronger predictor of $\mathrm{CV}$ death than renal failure [Mann et al. 2001; Adler et al. 2003]. Ang II may contribute to renal disease progression; local expression of the RAAS and production of Ang II, especially in diabetic renal tissues, may also promote the development and progression of renal damage. Although several studies have shown that more complete blockade of RAAS through ACEIs and ARBs may offer more effective cardiorenal protection, they may be not so effective because of alternative pathways [Cooper, 2004]. In particular, Ang II can be generated through a number of pathways besides the classic system. Renin is still the key enzyme in angiotensin peptide generation and seems to be the only route to Ang I formation. DRIs may have some advantages in terms of specificity by blocking Ang I generation. So direct renin inhibition would be a logical approach to achieving complete blockade of RAAS activity. 


\section{Direct renin inhibition in the hypertensive diabetic patient}

\section{Aliskiren in monotherapy versus ARBs}

In some recent important trials, aliskiren has also been shown renoprotective potential effect in patients with T2DM and albuminuria [Persson et al. 2008; Riccioni et al. 2010], the best available surrogate parameter in the treatment of diabetic nephropathy, associated with risk of renal and cardiovascular events [Fox et al. 2012].

In the Aliskiren in the Evaluation of Proteinuria in Diabetes (AVOID) trial, Parving and colleagues evaluated the effects of dual blockade of the RAAS with aliskiren and losartan in 599 hypertensive T2DM patients with nephropathy [Parving et al. 2008]. Patients were maintained on losartan (100 $\mathrm{mg}$ daily) for the duration of the study and were randomized to receive a 6 -month treatment with aliskiren ( $150 \mathrm{mg} /$ daily for 3 months, then $300 \mathrm{mg}$ / daily for 3 months) or placebo. After 3 months of treatment with aliskiren $(150 \mathrm{mg} /$ daily $)$, the urinary albumin:creatinine ratio (UACR) had decreased by $11 \%$ compared with placebo. Increasing the dose of aliskiren to $300 \mathrm{mg} /$ daily caused a further decrease in the UACR to $20 \%$. A nonsignificant difference in $\mathrm{BP}$ was seen between the treatment groups, suggesting that the renoprotective effect of aliskiren was independent of BP. There was no difference in the rates of adverse events, such as hyperkalemia, or discontinuation rates between the two groups.

In a subsequent double-blind, randomized, crossover study, 26 patients with T2DM, hypertension, and albuminuria ( $>100 \mathrm{mg} /$ day) were randomly assigned to four 2-month treatment periods in random order with placebo, $300 \mathrm{mg}$ aliskiren once daily, $300 \mathrm{mg}$ irbesartan once daily, or the combination of two drugs in identical doses. In this study Persson and colleagues demonstrated that with the combination of $300 \mathrm{mg}$ aliskiren and $300 \mathrm{mg}$ irbesartan, the reduction in albuminuria was enhanced compared with the reduction obtained with the two monotherapies [Persson et al. 2009]. The added antiproteinuric effect with combination treatment compared with aliskiren alone was $31 \%$. These two studies suggest that aliskiren plus an ARB may provide cardiorenal benefits that go beyond those solely attributable to lowering of BP.

\section{Aliskiren in combination with ACEls and ARBs}

Although in clinical studies aliskiren has been proved to reduce proteinuria, the early termination of the Aliskiren Trial in Type 2 Diabetes Using Cardiovascular and Renal Disease Endpoints (ALTITUDE) confirms previous concerns about the full suppression of the RAAS, in this case with aliskiren combined with an ACEI or ARB, in patients with diabetes and concomitant renal impairment [Parving et al. 2012]. This study was undertaken to determine whether use of the aliskiren would reduce cardiovascular and renal events (cardiovascular death, nonfatal myocardial infarction, nonfatal stroke, unplanned hospitalization for heart failure, end-stage renal disease, death attributable to kidney failure, the need for renalreplacement therapy with no dialysis or transplantation available or initiated, doubling of the baseline serum creatinine level) in patients with T2DM and chronic kidney disease (CKD), CVD, or both. In this double-blind trial 8561 patients were randomized to receive aliskiren (300 mg daily) or placebo as an adjunct to ACEI or ARB. The trial was stopped prematurely after the second interim efficacy analysis. The primary endpoint had occurred in 783 patients $(18.3 \%)$ assigned to aliskiren as compared with $732(17.1 \%)$ assigned to placebo. The effects on secondary renal end points were similar. Systolic and diastolic BPs were lower with aliskiren and the mean reduction in the urinary albumin-to-creatinine ratio was greater. In contrast the proportion of patients with hyperkalemia (serum potassium level $\geq 6 \mathrm{mmol} / \mathrm{l}$ ) was significantly higher in the aliskiren group than in the placebo group $(11.2 \%$ versus $7.2 \%)$, as was the proportion with reported hypotension $(12.1 \%$ versus $8.3 \%$ ). The authors concluded that the addition of aliskiren to standard therapy with RAAS in patients with T2DM who are at high risk for cardiovascular and renal events is not supported by these data and may even be harmful as a result of an increased incidence of serious adverse events in the aliskiren $300 \mathrm{mg}$ arm.

Recently, Wu and colleagues added aliskiren (150 $\mathrm{mg} /$ daily) for 6 months in 103 Chinese CKD patients who had been treated with ACEIs or ARBs and still had significant proteinuria or uncontrolled hypertension [Wu et al. 2012]. BP, serum creatinine, estimated glomerular filtration rate (eGFR), serum potassium, and spot urine UPCR were measured at 3 and 6 months after aliskiren add-on therapy and compared with baseline values. The combination of aliskiren and ACEI or ARB significantly reduced UPCR by $23 \%$ and mean arterial pressure at 6 months without significant changes in eGFR and serum potassium level. The authors conclude the addiction of 
$150 \mathrm{mg} /$ daily of aliskiren on ACEI or ARB in CKD patients, both in diabetes and nondiabetes, has a favorable effect on reducing residual proteinuria and inadequately controlled BP, with a good tolerability profile.

\section{Conclusion}

Some of the remaining questions regarding the action of aliskiren on cardiovascular and renal disorders are open. In particular, the combination therapy of aliskiren and RAAS blocker in diabetic hypertensive patients with CKD is controversial. Even if several published studies demonstrated that aliskiren is suitable for once-daily administration, its antihypertensive effect is comparable or superior to that of other antihypertensive agents at recommended doses with tolerability profile placebo-like at the licensed doses of 150 and $300 \mathrm{mg}$ daily. Currently, combination therapy of aliskiren with ACEIs and ARBs is not recommended in patients with T2DM and renal impairment. Pending disclosure of full results, the early termination of the ALTITUDE study seems to confirm previous concerns about the safety of the dual pharmacological blockade of the RAAS in these patients, even if in a recent published open-label study of low-dose aliskiren (150 mg/daily) in association with ACEIs or ARBs has demonstrated a good tolerability profile without the adverse events seen in ALTITUDE (hyperkalemia) [Wu et al. 2012].

The reasons for this discrepancy may be represented at least by two important aspects. First the dose of aliskiren used in the ALTITUDE trial (300 $\mathrm{mg}$ /daily) added to ACEIs and ARBs may be high. In fact, in the study by Wu and colleagues, even if it presents some limitations (open-label study without placebo, short therapy period), a lower dose of aliskiren (150 mg/daily) added to other standard therapies (ACEIs and ARB) has produced a good BP control without adverse events, in particular hyperkalemia [Wu et al. 2012]. Second, like ACEIs and ARBs, aliskiren increases plasma renin concentration, reduces PRA and has an additional ability to downregulate the expression of the prorenin receptor (PRR) and the $\mathrm{AT}(1)$ receptor, adding novel mechanistic insights to our current knowledge. The PRR is a receptor for renin and prorenin, not only allowing local production of Ang I from angiotensinogen, but also inducing intracellular signaling [Jagadeesh et al. 2012]. Recent studies implicate that deletion of PRR results in the dysfunction of V-ATPase, suggesting that the PRR is essential for its role as a proton pump with exchange of many electrolytes such as potassium [Wu et al. 2012]. Some questions remain unresolved, such as the potential effects of aliskiren on prorenin and its receptor-mediated Ang II-independent pathways, and the transduction network signals [Ichihara and Kinouchi, 2011].

\section{Funding}

This article received no specific grant from any funding agency in the public, commercial, or notfor-profit sectors.

\section{Conflict of interest statement}

The author declares that there are no conflicts of interest.

\section{References}

Adler, A., Stevens, R., Manley, S., Bilous, R., Cull, C., Holman, R. et al. (2003) Development and progression of nephropathy in type 2 diabetes: the United Kingdom Prospective Diabetes Study (UKPDS 64). Kidney Int 63: 225-232.

Angeli, F., Reboldi, G., Mazzotta, G., Poltronieri, C. and Verdecchia, P. (2012) Safety and efficacy of aliskiren in the treatment of hypertension: a systematic overview. Exp Opin Drug Saf 11: 659-670.

Anothaisintawee, T., Rattanasiri, S., Ingsathit, A., Attia, J. and Thakkinstian, A. (2009) Prevalence of chronic kidney disease: a systematic review and metaanalysis. Clin Nephrol 71: 244-254.

Bruckner A. (2007) Aliskiren (Tekturna $\left.{ }^{\circledR}\right)$ Novartis. Drug Monograph. Creighton University Drug Information Center. East Hanover, NJ: Novartis Pharmaceuticals Corporation.

Buse, J., Ginsberg, H., Bakris, G., Clark, N., Costa, F., Eckel, R. et al. (2007) Primary prevention of cardiovascular diseases in people with diabetes mellitus: a scientific statement from the American Heart Association and the American Diabetes Association. Circulation 115:

114-126.

Cagnoni, F., Njwe, C.A., Zaninelli, A., Ricci, A., Daffra, D., D'Ospina, A. et al. (2010) Blocking the RAAS at different levels: an update on the use of the direct renin inhibitors alone and in combination. Vasc Health Risk Manag 6: 549-559.

Campbell, D. (2008) Interpretation of plasma renin concentration in patients receiving aliskiren therapy. Hypertension 51: 15-18.

Cheng, J. (2008) Aliskiren: renin inhibitor for hypertension management. Clin Ther 30: 31-47. 
Cooper, M. (2004) The role of the renin-angiotensinaldosterone system in diabetes and its vascular complications. Am f Hypertens 17: 16S-20S.

Egan, B., Zhao, Y. and Axon, R. (2012) U.S. trends in prevalence, awareness, treatment, and control of hypertension, 1988-2008. F Am Med Assoc 303: 2043-2050.

Ferrannini, E. and Cushman, W. (2012) Diabetes and hypertension: the bad companions. Lancet 380 : 601-610.

Fox, C., Matsushita, K., Woodward, M., Bilo, H., Chalmers, J., Heerspink, H. et al. (2012) Associations of kidney disease measures with mortality and endstage renal disease in individuals with and without diabetes: a meta-analysis. Lancet 380: 1662-1673.

Giovanna, L., Francesca, V. and Roberto, P. (2012) RAAS inhibition and renal protection. Curr Pharm Des 18: 971-980.

Gross, J., de Azevedo, M., Silveiro, S., Canani, L., Caramori, M. and Zelmanovitz, T. (2005) Diabetic nephropathy: diagnosis, prevention, and treatment. Diabetes Care 28: 164-176.

Grossman, E. and Messerli, F. (2011) Management of blood pressure in patients with diabetes. $A m \mathcal{F}$ Hypertens 24: 863-875.

Hsueh, W. and Wyne, K. (2011) Renin-Angiotensinaldosterone system in diabetes and hypertension. $\mathcal{f}$ Clin Hypertens 13: 224-237.

Ichihara, A. and Kinouchi, K. (2011) Current knowledge of (pro)renin receptor as an accessory protein of vacuolar $\mathrm{H}+-\mathrm{ATPase} . \mathcal{F}$ Renin Angiotensin Aldosterone Syst 12: 638-640.

Jagadeesh, G., Balakumar, P. and Stockbridge, N. (2012) How well do aliskiren's purported mechanisms track its effects on cardiovascular and renal disorders? Cell Signal 24: 1583-1591.

Ma, J. and Stafford, R. (2008) Screening, treatment, and control of hypertension in U.S. private physician offices, 2003-2004. Hypertension 51: 1275-1281.

Mann, J., Gerstein, H., Pogue, J., Bosch, J. and Yusuf, S. (2001) Renal insufficiency as a predictor of cardiovascular outcomes and the impact of ramipril: the HOPE randomized trial. Ann Intern Med 34: 629-636.

Mentz, R., Bakris, G., Waeber, B., McMurray, J., Gheorghiade, M., Ruilope, L. et al. (2012) The past, present and future of renin-angiotensin aldosterone system inhibition. Int $\mathcal{F}$ Cardiol. DOI: 10.1016/j. ijcard.2012.10.007.

Oparil, S., Yarows, S., Patel, S., Fang, H., Zhang, J. and Satlin, A. (2007) Efficacy and safety of combined use of aliskiren and valsartan in patients with hypertension: a randomised, double-blind trial. Lancet 370: 221-229.

Parving, H., Persson, F., Lewis, J., Lewis, E. and Hollenberg, N. (2008) Aliskiren combined with losartan in type 2 diabetes and nephropathy. New Engl f Med 358: 2433-2446.

Parving, H., Brenner, B., McMurray, J., de Zeeuw, D., Haffner, S., Solomon, S. et al. (2012) Cardiorenal end points in a trial of aliskiren for type 2 diabetes. $N$ Engl f Med 367: 2204-2213.

Perk, J., De Backer, G., Gohlke, H., Graham, I., Reiner, Z., Verschuren, M. et al. (2012) European Guidelines on cardiovascular disease prevention in clinical practice (version 2012). The Fifth Joint Task Force of the European Society of Cardiology and Other Societies on Cardiovascular Disease Prevention in Clinical Practice. Developed with the special contribution of the European Association for Cardiovascular Prevention \& Rehabilitation (EACPR). Eur Heart f 33: 1635-1701.

Persson, F., Rossing, P., Schjoedt, K., Juhl, T., Tarnow, L., Stehouwer, C. et al. (2008) Time course of the antiproteinuric and antihypertensive effects of direct renin inhibition in type 2 diabetes. Kidney Int 73: 1419-1425.

Persson, F., Rossing, P., Reinhard, H., Juhl, T., Stehouwer, C., Schalkwijk, C. et al. (2009) Renal effects of aliskiren compared to and in combination with irbesartan in patients with type 2 diabetes, hypertension and albuminuria. Diabetes Care 210: 1873-1879.

Rahuel, J., Rasetti, V., Maibaum, J, Rüeger, H., Göschke, R., Cohen, N. et al. (2000) Structure-based drug design: the discovery of novel non peptide orally active inhibitors of human renin. Chem Biol 7: 493-504.

Riccioni, G. (2011) Aliskiren in the treatment of hypertension and organ damage. Cardiovasc Ther 29: 77-87.

Riccioni, G., Vitulano, N., D’Orazio, N. and Bellocci, F. (2009) Aliskiren, the first approved renin inhibitor: Clinical application and safety in the treatment of hypertension. Adv Ther 26: 700-710.

Riccioni, G., Vitulano, N., Zanasi, A., Bellocci, F. and D'Orazio, N. (2010) Aliskiren: beyond blood pressure reduction. Expert Opin Investig Drugs 19: 1265-1274.

Sowers, J., Epstein, M. and Frolich, E. (2001) Diabetes, hypertension, and cardiovascular disease. An update. Hypertension 37: 1053-1059.

Vaidyanathan, S., Jermany, J., Yeh, C., Bizot, M. and Camisasca, R. (2006) Aliskiren, a novel orally effective renin inhibitor, exhibits similar pharmacokinetics and pharmacodynamics in Japanese 
and Caucasian subjects. Br $\mathcal{F}$ Clin Pharmacol 62: 690-698.

Volpe, M., Cosentino, F., Tocci, G., Palano, F. and Paneni, F. (2011) Antihypertensive therapy in diabetes: the legacy effect and RAAS blockade. Curr Hypertens Rep 4: 318-324.

Wu, M., Tung, S., Hsu, K. and Lee, C. (2012) Aliskiren add-on therapy effectively reduces proteinuria in chronic kidney disease: An open-label prospective trial. F Renin Angiotensin Aldosterone Syst, in press.

Von Lueder, T. and Krum, H. (2012) RAAS inhibitors and cardiovascular protection in large scale trials. Cardiovasc Drugs Ther 2012 Dec 7.
Waldmeier, F., Glaenzel, U., Wirtz, B., Oberer, L., Schmid, D., Seiberling, M. et al. (2007) Absorption, distribution, metabolism and elimination of direct renin inhibitor aliskiren in healthy volunteers. Drug Metab Dispos 35:

1418-1428.

Wu, M., Tung, S., Hsu, K. and Lee, C. (2012) Aliskiren add-on therapy effectively reduces proteinuria in chronic kidney disease: An open-label prospective trial. F Renin Angiotensin Aldosterone Syst. PMID: 23223162.

Wuerzner, G. and Azizi, M. (2008) Renin inhibition with aliskiren. Clin Exp Pharmacol Physiol 35:

426-430.
Visit SAGE journals online http://tae.sagepub.com

(SAGE journals 